\title{
Antibody Response to SARS-CoV-2 Vaccination in Patients with Inflammatory Bowel Disease: Results of a Single-Center Cohort Study in a Tertiary Hospital in Germany
}

\author{
Johanna Maria Classen Anna Muzalyova Sandra Nagl Carola Fleischmann \\ Alanna Ebigbo Christoph Römmele Helmut Messmann Elisabeth Schnoy \\ Internal Medicine III, University Hospital Augsburg, Augsburg, Germany
}

\begin{abstract}
Keywords
Inflammatory bowel disease - Crohn's disease - Ulcerative colitis - Severe acute respiratory syndrome corona virus-2 . Vaccination
\end{abstract}

\begin{abstract}
Background: COVID-19 is a viral disease caused by severe acute respiratory syndrome corona virus 2 (SARS-CoV-2), first described in 2019, with a significant impact on everyday life since then. In December 2020, the first vaccine against COVID-19 from BioNTech/Pfizer was approved for the first time. However, little is known about the immune response to vaccination in patients with inflammatory bowel disease (IBD) and immunomodulators or biologics. The aim of our study was to investigate antibody response to SARS-CoV-2 vaccination in patients with IBD receiving immunomodulators or biologics compared to healthy controls. Methods: This was a single-center study with a retrospective observational design. Seventy-two patients with ulcerative colitis or Crohn's disease were included. Matching data from 72 healthy employees of our hospital were used as the control group. Data were matched by propensity score to patients with IBD. Blood samples were taken from both groups for antibody response, and both groups received an accompanying questionnaire. Results: Sixty-five (90.3\%) patients of
\end{abstract}

the IBD group reported taking immunomodulatory therapy. The mean antibody level for all IBD patients was 1,257.1 U/ $\mathrm{mL}$ (standard deviation [SD] 1,109.626) in males and 1,500.1 $\mathrm{U} / \mathrm{mL}$ (SD 1142.760) in female IBD patients after full vaccination. Compared to the healthy group, reduced antibody response could be detected (IBD group 1,383.76 U/mL SD 1,125.617; control group 1,885.65 U/mL SD 727.572, $p<$ $0.05)$. In this group, blood samples were taken with an average of 61.9 days after the first vaccination. There was no vaccination failure in the IBD group after 2 vaccinations. After the first vaccination, side effects, including muscle pain, pain at the injection site, and fatigue, were reported more often in IBD patients than in the control group (total symptoms IBD group 58.3\%, control group $34.5 \%, p<0.007$ ). The opposite occurred after the second vaccination when side effects were higher in the control group (total symptoms IBD group $55.4 \%$, control group $76 \%, p=0.077$ ). There was a trend to a reduced immune response in elderly patients. Disease duration and concomitant immunomodulatory therapy (TNF-alpha blockers, interleukin inhibitors, integrin inhibitors, methotrexate, or azathioprine) had no impact on the immune response. However, longer time to last medication given and time passed to vaccination in patients with IBD seems to have a positive impact on antibody levels. Conclusion: Overall, we could show a high antibody response to vaccination with COVID-19 in all patients with IBD after 2 karger@karger.com

www.karger.com/ddi

(c) 2021 S. Karger AG, Base

Karger"
Correspondence to:

Johanna Maria Classen, johanna-maria.classen@uk-augsburg.de Elisabeth Schnoy, elisabeth.schnoy@uk-augsburg.de 
vaccinations. Vaccination was well tolerated, and no other adverse events were detected. Concomitant immunomodulatory therapy (TNF-alpha blockers, interleukin inhibitors, integrin inhibitors, methotrexate, or azathioprine) had no impact on seroconversion. Further evaluation of antibody titers over time is mandatory to detect early the need for re-vaccination in these patients.

(c) 2021 S. Karger AG, Basel

\section{Introduction}

COVID-19 is a viral disease caused by severe acute respiratory syndrome corona virus 2 (SARS-CoV-2), first described in 2019 [1]. Currently, more than 3.8 million cumulative patients in Germany suffer from SARS-CoV-2 [2]. Rapid development of vaccination against COVID-19 was a key challenge in the resolution of the COVID-19 pandemic. In December 2020, the first vaccination against COVID-19 from BioNTech/Pfizer was approved [3], followed by Moderna and AstraZeneca vaccines [4]. Several further vaccines have been approved or are on the way [5].

The risk of COVID-19 infection in patients with inflammatory bowel disease (IBD), including Crohn's disease (CD) and ulcerative colitis (UC) is similar to the general population [6-9]. Although there are clear recommendations for vaccination against SARS-CoV-2 in IBD patients [10-12], knowledge about seroconversion rates and response in IBD patients, especially with immunomodulatory therapies or biologics, are scarce. These patient cohorts were excluded in the pivotal trials of COVID-19 vaccinations, and published data are still rare [13]. In this single-center cohort study, we aimed to define antibody response to SARS-CoV-2 vaccination in patients with IBD receiving immunomodulators or biological therapies compared to healthy controls.

\section{Materials and Methods}

\section{Study Population}

This was a single-center study with a retrospective observational design. The study was performed at the University Hospital Augsburg, Germany, in accordance with the Declaration of Helsinki and Good Clinical Practice.

Seventy-two patients with IBD (IBD group) at our outpatient clinic were consecutively included in our study and in our COVID-19 registry (COKA registry). Data from 72 healthy employees (control group) included in the COVID-19 registry of University Hospital Augsburg (COKA registry) were used as a control group and matched with propensity scores to patients with IBD. The
COKA registry aims at collecting data regarding infection with SARS-CoV-2 and COVID-19 vaccinations. The collection of data on vaccination from employees of our hospital is performed voluntarily and pseudonymized.

Data on vaccinated employees analyzed in this study were included in the COKA registry between January 01, 2021 and May 19, 2021; data on vaccinated IBD patients were collected between May 19, 2021, and June 24, 2021. A standardized blood sample was collected from employees and patients with IBD and analyzed on the same day.

In addition, both groups received an accompanying questionnaire (online suppl. 1, 2; for all online suppl. material, see www.karger.com/doi/10.1159/000521343) containing information, e.g, on age, sex, immunosuppressive medication, chronic diseases or immunodeficiency, allergies, and clinical symptoms after vaccination.

All included employees received both vaccinations with BNT162b2 vaccine from BioNTech/Pfizer, with the second dose administered 3 weeks after the initial vaccination. Employees included in our study had no chronic diseases or any immunomodulatory therapies.

IBD patients received various vaccine combinations, with the majority being administered with the same vaccine, such as BNT162b2 vaccine from BioNTech/Pfizer, Moderna, or AstraZeneca upon first and second vaccination. However, due to changing recommendations of the "Ständige Impfkomission" (STIKO) from the Robert-Koch Institute in Germany, some patients receiving vaccination with the AstraZeneca vaccine upon first vaccination were administered with a second dose of BioNTech/Pfizer or Moderna vaccine. IBD patients were treated with different immunosuppressive therapies such as TNF-alpha blockers, interleukin inhibitors, integrin inhibitors, methotrexate, or azathioprine. The primary purpose was to analyze the early antibody response for patients with IBD with or without immunomodulators or biologics compared to healthy controls after 2 vaccinations.

\section{Test Method}

The collected serum samples were tested in the in-house laboratory for the presence of SARS-CoV-2 antibodies (IgG) against the receptor-binding domain of the spike protein (S) using immunoassays Elecsys ${ }^{\circledR}$ Anti-SARS-CoV-2S (Roche Diagnostics, Germany). In brief, the quantitative result (sensitivity $99.85 \%$, specificity $99.98 \%$ linear measuring range is between 0.4 and 2,500 U/ $\mathrm{mL}$ ) was evaluated according to the manufacturer's specifications.

\section{Statistical Analysis}

For comparison of response to vaccination and seroconversion between patients with IBD and healthy controls, propensity scores were used to create a cohort of matched healthy study participants with equal distribution of baseline characteristics such as gender, age, days since first vaccination, and history of reduced immune response to previous vaccinations (IBD patients compared to healthy employees).

Data were initially analyzed descriptively with categorical variables presented as absolute frequencies and percentages and continuous variables described with means and standard deviations [SDs]. Dependencies between categorical variables were analyzed using the $\chi^{2}$ test. A comparison of means was conducted using the Mann-Whitney-U test. The relationship between categorically scaled variables was analyzed, applying Spearman's rank correlation. 
Table 1. General demographics of IBD and control group and vaccination types applied at first and second vaccination in IBD patients

\begin{tabular}{|c|c|c|c|c|c|}
\hline & \multicolumn{2}{|l|}{ IBD group } & \multicolumn{2}{|l|}{ Control group } & \multirow[t]{2}{*}{$p$ value } \\
\hline & $n$ & $\%$ & $n$ & $\%$ & \\
\hline \multicolumn{6}{|l|}{ Sex } \\
\hline Male & 34 & 47.2 & 35 & 48.6 & \multirow{2}{*}{0.868} \\
\hline Female & 38 & 52.8 & 37 & 51.4 & \\
\hline Age (mean) & \multicolumn{2}{|l|}{$48.4(S D=15.236)$} & \multicolumn{2}{|l|}{$46.3(\mathrm{SD}=12.460)$} & 0.371 \\
\hline Days since vaccination & \multicolumn{2}{|l|}{$56.4(\mathrm{SD}=31.485)$} & \multicolumn{2}{|l|}{$61.9(S D=26.064)$} & 0.257 \\
\hline \multicolumn{6}{|l|}{ 1st vaccination } \\
\hline BioNTech/Pfizer & 59 & 81.9 & 72 & 100.0 & \multirow{3}{*}{0.001} \\
\hline Moderna & 2 & 2.8 & 0 & 0.0 & \\
\hline AstraZeneca & 11 & 15.3 & 0 & 0.0 & \\
\hline \multicolumn{6}{|l|}{ 2nd vaccination } \\
\hline BioNTech/Pfizer & 64 & 92.6 & 72 & 100.0 & \multirow{3}{*}{0.064} \\
\hline Moderna & 4 & 5.9 & 0 & 0.0 & \\
\hline AstraZeneca & 1 & 1.5 & 0 & 0.0 & \\
\hline \multicolumn{6}{|l|}{ Several vaccinations } \\
\hline No & 59 & 81.9 & 62 & 86.1 & \multirow{3}{*}{0.515} \\
\hline Yes & 4 & 5.6 & 5 & 6.9 & \\
\hline Unknown & 9 & 12.5 & 5 & 6.9 & \\
\hline
\end{tabular}

The significance level was defined at $p<0.05$. $p$ value of 0.05 was regarded as significant, a $p$ value of 0.01 was regarded as highly significant. Data management, descriptive, and inference-statistical analysis were conducted using IBM SPSS Version 27.

\section{Results}

\section{General Patient Demographics}

Out of 72 patients included in the IBD group, $47.2 \%$ were male. Seventy-two patients in the control group (48.6\% male) were included. In IBD patients, the mean age was 48.4 (SD 15.236) years and 46.3 (SD 12.460) years in the control group. Days since the last vaccination were similar in both groups: 56.4 SD 31.485 days in IBD group, 61.9 SD 26.064 in the control group. Overall, no significant differences were detected between both groups concerning baseline characteristics such as sex $(p=0.686)$, age $(p=0.371)$, and days since last vaccination $(p=0.257)$ or history of reduced immune response to previous vaccinations requiring additional doses $(p=0.515)$. The type of vaccination applied differed significantly between both examined groups $(p<0.001$ first vaccination AstraZene$c a)$. All included employees received both vaccinations with BNT162b2 vaccine from BioNTech/Pfizer, with the second dose administered on the 21st day after the initial vaccination.

Crohn's Disease, Ulcerative Colitis, SARS-

CoV-2, and Vaccination
IBD group received various vaccine combinations, with the majority being administered with the same vaccine, such as BNT162b2 vaccine from BioNTech/Pfizer, Moderna, or AstraZeneca upon first and second vaccination. However, due to changing recommendations of the STIKO, some patients who had initially received the AstraZeneca vaccine, were subsequently vaccinated a second time with the BioNTech/Pfizer or Moderna vaccine. Seven patients received different vaccines upon first and second vaccination. Fifty-nine patients $(81.9 \%)$ received the first vaccination with BioNTech/Pfizer, 11 (15.3\%) with AstraZeneca, and 2 patients with Moderna (2.8\%). Sixty-four patients (92.6\%) underwent the second vaccination with BioNTech/Pfizer, 4 patients (5.9\%) with Moderna, and 1 patient (1.5\%) with AstraZeneca vaccine. Patient demographics are given in detail in Table 1.

\section{Clinical Characteristics of Patients with IBD}

In patients with IBD, 40 patients had CD (55.6\%) and $32(44.4 \%)$ patients had UC. The mean disease duration was 13.01 (SD 10.613) years. Three patients suffered from SARS-CoV-2 infection, in all 3 cases asymptomatic (4.2\%); 69 patients were not infected (95.8\%). Sixty-five (90.3\%) patients of the IBD group reported taking immunomodulatory treatment. The remaining 7 (9.7\%) were not under permanent immunomodulatory therapy. Most of the patients $(27,37.5 \%)$ were treated with TNF-alpha 
Table 2. Patient characteristics of IBD group concerning current medical treatment, application intervals, and mode of application

\begin{tabular}{|c|c|c|c|}
\hline Category & Dimension & $n$ & $\%$ \\
\hline \multirow[t]{2}{*}{ IBD } & $C D$ & 40 & 55.6 \\
\hline & UC & 32 & 44.4 \\
\hline \multicolumn{2}{|l|}{ Duration of IBD } & \multicolumn{2}{|l|}{$13.01(\mathrm{SD}=10.613)$} \\
\hline \multirow[t]{2}{*}{ COVID-19 infection } & Yes & 3 & 4.2 \\
\hline & No & 69 & 95.8 \\
\hline \multirow[t]{11}{*}{ Treatment } & Immunosuppression total & 65 & 90.3 \\
\hline & Steroids & 2 & 2.8 \\
\hline & Mesalazine & 24 & 33.3 \\
\hline & Azathioprine & 1 & 1.4 \\
\hline & Methothrexate & 1 & 1.4 \\
\hline & Calcineurin inhibitor & 0 & 0.0 \\
\hline & TNF blocker & 27 & 37.5 \\
\hline & Integrin inhibitor & 19 & 26.4 \\
\hline & JAK inhibitor & 0 & 0.0 \\
\hline & Ustekinumab & 14 & 19.4 \\
\hline & Other therapies & 0 & 0.0 \\
\hline \multirow[t]{8}{*}{ Application interval in weeks } & 0 & 1 & 1.6 \\
\hline & 1 & 2 & 3.2 \\
\hline & 2 & 9 & 14.5 \\
\hline & 4 & 5 & 8.1 \\
\hline & 5 & 2 & 3.2 \\
\hline & 6 & 6 & 9.7 \\
\hline & 7 & 1 & 1.6 \\
\hline & 8 & 36 & 58.1 \\
\hline \multirow[t]{3}{*}{ Mode of application } & IV & 34 & 47.2 \\
\hline & p.o & 1 & 1.4 \\
\hline & S.C & 22 & 30.6 \\
\hline
\end{tabular}

blockers. Integrin inhibitors such as vedolizumab constituted the long-term therapy of 19 (26.4\%) patients. Fourteen (19.4\%) patients reported taking interleukin inhibitors (ustekinumab). One patient reported (1.4\%) taking methotrexate or azathioprine, respectively. Calcineurin inhibitors, JAK inhibitors, or other therapies were not applied in any therapy of included IBD patients. Only $2(2.8 \%)$ patients received steroid medication at the time of blood sampling (1 patient with adrenal insufficiency and 1 patient with an acute IBD flare; low-dose steroids were given in both patients). Twenty-four patients (33.3\%) had a concomitant therapy which included mesalazine.

Patients also indicated the interval at which immunosuppressive therapy was administered. Most of the patients received their therapy every 8 weeks $(n=36,58.1 \%)$, 14 of them were taking interleukin inhibitors, 11 each took TNF-alpha blockers or integrin inhibitors. One of the patients with a TNF-alpha blocker therapy every 8 weeks received additional azathioprine every 8 weeks. Seven week-intervals were reported by 1 patient $(1.6 \%)$ in his use of a TNF-alpha blocker. An interval of 6 weeks was reported by 6 patients (9.7\%), 2 of them were taking integrin inhibitors, and 4 TNF-alpha blockers. Five weeks were reported by $2(3.2 \%)$ patients, both were taking TNF-alpha blockers. A 4-week interval was reported in 5 patients $(8.1 \%)-3$ of them were taking TNF-alpha blockers and 2 of them integrin inhibitors. Nine IBD patients (14.5\%) had an application interval of 2 weeks, of which 5 patients received TNF-alpha blockers and 4 patients received integrin inhibitors. Two (3.2\%) had only 1 week between the doses - 1 patient was under a therapy with methotrexate, and 1 was receiving a TNF-alpha blocker. In 34 patients (47.2\%), immunosuppressive therapy was administered intravenously. Twenty-two (30.6\%) patients received their therapy subcutaneously. Only $1(1.4 \%)$ had an oral substitution. Details are listed in Table 2. 
Table 3. Reported side effects after first and second vaccination in IBD and control group

\begin{tabular}{|c|c|c|c|c|c|}
\hline & \multicolumn{2}{|c|}{ IBD group } & \multicolumn{2}{|c|}{ Control group } & \multirow[t]{2}{*}{$p$ value } \\
\hline & $n$ & $\%$ & $n$ & $\%$ & \\
\hline \multicolumn{6}{|c|}{ Symptoms 1st vaccination } \\
\hline Total of symptoms & 42 & 58.3 & 20 & 34.5 & 0.007 \\
\hline Muscle pain & 15 & 20.8 & 6 & 16.7 & 0.606 \\
\hline Fever & 3 & 4.2 & 1 & 2.8 & 1.000 \\
\hline Joint pain & 7 & 9.9 & 4 & 11.1 & 1.000 \\
\hline Local redness & 4 & 5.6 & 0 & 0.0 & 0.299 \\
\hline Pain injection side & 31 & 43.1 & 15 & 41.7 & 0.891 \\
\hline Fatigue & 22 & 30.6 & 5 & 14.3 & 0.096 \\
\hline Nausea/vomiting & 1 & 1.4 & 1 & 2.8 & 1.000 \\
\hline Diarrhea & 2 & 2.8 & 0 & 0.0 & 0.551 \\
\hline \multicolumn{6}{|c|}{ Symptoms 2nd vaccination } \\
\hline Total of symptoms & 31 & 55.4 & 19 & 76.0 & 0.077 \\
\hline Muscle pain & 9 & 16.1 & 7 & 28.0 & 0.213 \\
\hline Fever & 5 & 8.9 & 5 & 20.0 & 0.162 \\
\hline Joint pain & 6 & 10.7 & 10 & 40.0 & 0.004 \\
\hline Local redness & 0 & 0.0 & 2 & 8.0 & 0.093 \\
\hline Pain injection side & 14 & 25.0 & 12 & 48.0 & 0.041 \\
\hline Fatigue & 20 & 35.7 & 10 & 40.0 & 0.712 \\
\hline Nausea/vomiting & 0 & 0.0 & 2 & 8.0 & 0.093 \\
\hline Diarrhea & 1 & 1.8 & 1 & 4.0 & 1.000 \\
\hline
\end{tabular}

\section{Association of Immune Response with Medical Therapies}

All patients with IBD developed an immune response after full vaccination. The mean antibody titer accounted for $1,257.1 \mathrm{U} / \mathrm{mL}(\mathrm{SD} 1,109.626)$ in male patients and $1,500.15 \mathrm{U} / \mathrm{mL}$ (SD 1,142.760) in female patients $(p=0.419)$.

Overall, there was no significant difference in antibody levels between the 3 different vaccines received upon first vaccination $(p=0.904)$. The highest antibody titer after the first vaccination was detected in the group receiving the AstraZeneca vaccine with $1,432.16 \mathrm{U} / \mathrm{mL}$ (SD 1,236.874), followed by BioNTech/Pfizer vaccine with $1,375.76 \mathrm{U} / \mathrm{mL}$ (SD 1,113.815) and Moderna with 1,349.50 $\mathrm{U} / \mathrm{mL}$ (SD 1,627.053) antibody titer levels. Regarding the second vaccination, the antibody level was higher in the group administered with Moderna accounting for 1,924.75 U/mL (SD 1,150.500) followed by BioNTech/ Pfizer with 1,434.62 U/mL (SD 1,108.420). In 1 person receiving AstraZeneca as the second vaccine, an antibody level of $48.2 \mathrm{U} / \mathrm{mL}$ was detected. The vaccine administered the second time did not influence the antibody levels ( $p$ value 0.332 ). Patients who received different vaccines upon first and second vaccination showed a substantially higher antibody level of 2,226.71 U/mL (SD 723.046) compared to the group who received the same vaccine twice $(1,353.14 \mathrm{U} / \mathrm{mL}$ [SD 1,118.728]), this difference was not significant $(p=0.181)$. 
Table 4. Antibody response (in $\mathrm{U} / \mathrm{mL}$ ) in IBD group, depending on different medication given

\begin{tabular}{|c|c|c|c|}
\hline IBD group & Mean & SD & $p$ value \\
\hline \multicolumn{4}{|l|}{ Sex } \\
\hline Male & $1,257.10$ & $1,109.626$ & \multirow{2}{*}{0.419} \\
\hline Female & $1,500.15$ & $1,142.760$ & \\
\hline \multicolumn{4}{|l|}{ 1st vaccination } \\
\hline BioNTech/Pfizer & $1,375.76$ & $1,113.815$ & \multirow{3}{*}{0.904} \\
\hline Moderna & $1,349.50$ & $1,627.053$ & \\
\hline AstraZeneca & $1,432.16$ & $1,236.874$ & \\
\hline \multicolumn{4}{|l|}{ 2nd vaccination } \\
\hline BioNTech/Pfizer & $1,434.62$ & $1,108.420$ & \multirow{3}{*}{0.332} \\
\hline Moderna & $1,924.75$ & $1,150.500$ & \\
\hline AstraZeneca & 48.20 & & \\
\hline \multicolumn{4}{|l|}{ Different vaccines } \\
\hline No & $1,353.14$ & $1,118.728$ & \multirow{2}{*}{0.181} \\
\hline Yes & $1,226.71$ & 723.046 & \\
\hline \multicolumn{4}{|c|}{ Need for several doses in earlier vaccination } \\
\hline No & $1,387.63$ & $1,136.033$ & \multirow{3}{*}{0.939} \\
\hline Yes & $1,420.4$ & $1,262.718$ & \\
\hline Unknown & $1,342.56$ & $1,135.979$ & \\
\hline \multicolumn{4}{|l|}{ IBD } \\
\hline$C D$ & $1,349.90$ & $1,100.590$ & \multirow{2}{*}{0.417} \\
\hline UC & $1,425.02$ & $1,171.744$ & \\
\hline \multicolumn{4}{|l|}{ Total } \\
\hline No & $1,203.64$ & $1,243.153$ & \multirow{2}{*}{0.513} \\
\hline Yes & $1,403.46$ & $1,120.987$ & \\
\hline \multicolumn{4}{|l|}{ Mesalazine } \\
\hline No & $1,386.61$ & $1,120.415$ & \multirow{2}{*}{0.830} \\
\hline Yes & $1,378.18$ & $1,159.923$ & \\
\hline \multicolumn{4}{|l|}{ TNF-alpha blockers } \\
\hline No & $1,554.28$ & $1,138.505$ & \multirow{2}{*}{0.063} \\
\hline Yes & $1,105.88$ & $1,066.817$ & \\
\hline \multicolumn{4}{|l|}{ Integrin inhibitors } \\
\hline No & $1,295.74$ & $1,104.166$ & \multirow{2}{*}{0.163} \\
\hline Yes & $1,624.66$ & $1,178.682$ & \\
\hline \multicolumn{4}{|l|}{ Interleukin inhibitors } \\
\hline No & $1,320.25$ & $1,125.969$ & \multirow{2}{*}{0.335} \\
\hline Yes & $1,642.32$ & $1,127.557$ & \\
\hline Total & $1,383.76$ & $1,125.617$ & \\
\hline
\end{tabular}

The kind of IBD disease had no significant effect on the level of antibody titers $(p=0.417)$. The same was observed for the type of medication, such as mesalazine $(1,378.18 \mathrm{U} / \mathrm{mL}$, SD $1,159.924, p=0.830)$, TNF inhibitors $(1,105.88 \mathrm{U} / \mathrm{mL}, \mathrm{SD} 1,066.817, p=0.063)$, integrin inhibitors $(1,624.66 \mathrm{U} / \mathrm{mL}$ SD 1,178.682, $p=0.335)$, and IL-12/IL-23 antibody $(1,642.32 \mathrm{U} / \mathrm{mL}$ SD $1,127.557, p=$ 0.335 ) (Table 4). IBD patients needing several doses in prior vaccinations had similar antibody levels compared to IBD patients with no need for re-vaccination $(1,420.4$ $\mathrm{U} / \mathrm{mL}$ vs. $1,387.63 \mathrm{U} / \mathrm{mL}, p<0.939)$.
Table 5. Antibody response (in $\mathrm{U} / \mathrm{mL}$ ) in control group

\begin{tabular}{llll}
\hline Control group & Mean & SD & $p$ value \\
\hline Sex & \multicolumn{4}{c}{} \\
$\quad$ Male & $1,257.10$ & $1,109.626$ & \\
$\quad$ Female & $1,500.15$ & $1,142.760$ & 0.415 \\
Need for several doses in earlier vaccination & & \\
$\quad$ No & $1,899.42$ & 724.826 & \\
$\quad$ Yes & $1,325.00$ & 739.268 & 0.339 \\
$\quad$ Unknown & $2,275.60$ & 501.774 & \\
\hline Total control group & $1,885.65$ & 727.572 & 0.05 \\
\hline
\end{tabular}

In the control group, the mean antibody titer was $1,257.10 \mathrm{U} / \mathrm{mL}$ (SD 1,109.626) in male participants; in females, the mean was $1,500.15 \mathrm{U} / \mathrm{mL}$ (SD 1,142.760, $p$ value 0.415$)$. Employees with no repeated vaccination doses in prior vaccinations had numerically higher antibody titers $(1,899.42 \mathrm{U} / \mathrm{mL}$ SD 724.826$)$ compared to employees with the need for several doses in prior vaccinations $(1,325.00 \mathrm{U} / \mathrm{mL}, \mathrm{SD} 739.268$, $p$ value 0.339$)$ (Table 5).

\section{Lower Antibody Titer in Elderly Patients}

A trend toward lower antibody titers in elderly patients was observed in both examined groups ( $p=0.230 \mathrm{IBD}$ and $p=0.177)$. However, these associations were not statistically significant $(0.1<p>0.05)$. There was a significant correlation between antibody response and time or days since the first vaccination: in patients with IBD, antibody titers were positively associated with days between last vaccination and blood sample taken $(\rho=0.387, p<0.01)$, whereas in the control group, antibody titers negatively correlated with the days after the first vaccination ( $\rho=$ $-0.287, p<0.05$ ). The days between vaccination 1 and 2 had no impact on antibody response in both groups. Furthermore, in IBD group the antibody titer level was positively associated with a longer time interval of the last medication given, the longer it had been since the last medication intake, the higher the level of antibody titers were. The application intervals were between 1 and 8 weeks (online suppl. Table 1$)$. However, this association was marginally not significant $(\rho=0.221,0.1<p>0.05)$. Disease duration had no impact on antibody response (Table 6).

\section{Discussion}

Our study cohort could demonstrate a comparable antibody response to SARS-CoV-2 vaccination after full vaccination in all patients with IBD compared to healthy 
Table 6. Correlation of antibody response in IBD and control group to age, days since vaccination, days between vaccination, disease duration, and last medication given

\begin{tabular}{|c|c|c|c|c|c|}
\hline & Age & $\begin{array}{l}\text { Days since } \\
\text { vaccination }\end{array}$ & $\begin{array}{l}\text { Days between } \\
\text { vaccinations }\end{array}$ & $\begin{array}{l}\text { Disease } \\
\text { duration }\end{array}$ & $\begin{array}{l}\text { Time between } \\
\text { last medication } \\
\text { given and } \\
\text { vaccination }\end{array}$ \\
\hline IBD group & $-0.230^{\diamond}$ & $0.387^{* *}$ & 0.063 & 0.038 & $0.221^{\diamond}$ \\
\hline Control group & $-0.177^{\diamond}$ & $-0.287^{*}$ & -0.106 & $\mathrm{X}$ & $\mathrm{X}$ \\
\hline
\end{tabular}

Level of significance. ${ }^{* *} p<0.01 .^{*} p<0.05 .{ }^{\diamond} 0.1>p>0.05$. controls. Although there was a significant difference in antibody response in patients treated with various immunosuppressive therapies or biologics compared to controls (IBD: 1,383.76 [1,125.617] vs. control: 1,885.65 [SD $=727.572] p<0.05)$, all IBD patients developed an excellent antibody response while receiving a variety of immunomodulatory therapies.

So far, it was shown that patients treated with infliximab might have attenuated serological responses after an infection with SARS-CoV-2 compared to healthy controls [14]. Regarding antibody formation after vaccination against SARS-CoV-2 in patients with infliximab, little is known. But it could have been shown that when additional immunosuppressant medication was used, the responses were even lower [15]. This aspect was observed in other vaccinations, e.g., pneumococcal vaccination in patients with IBD [16]. In 96 patients taking infliximab or a combination immunosuppressive therapy, response to this vaccination was significantly lower than control patients receiving mesalazine exclusively [16]. In addition, patients with IBD seem to have an impaired immune response to influenza vaccination, especially when undergoing simultaneous treatment with infliximab or immunomodulators $[17,18]$.

Up to now, only a few studies have reported antibody responses to SARS-CoV-2 vaccination in patients with IBD. In one study, 27 patients were monitored after their second vaccination with SARS-CoV-2. Patients were either treated with infliximab or vedolizumab. After their second vaccination, $85 \%(17 / 20)$ with infliximab treatment and $86 \%(6 / 7)$ with vedolizumab treatment experienced a seroconversion [15]. A recently published study included a total of 12,231 patients with IBD. In both groups (CD and UC), vaccination with BNT162b2 mRNA COVID-19 vaccine was highly effective [19]. Despite this huge patient cohort of 12,231 patients, only for a minority of these patients included, detailed information about the IBD treatment applied was provided (12 patients with 5-ASA, 3 patients with anti-TNF, 1 patient with methotrexate).

Another study with 48 patients showed a $100 \%$ seropositivity after the second vaccination. In this group,
BioNTech/Pfizer and Moderna were administered. However, lower antibody levels were found in patients with vedolizumab and partially for anti-TNFs in this cohort [20]. A recently published study with 28 patients demonstrated a SARS-CoV-2-antibody response in $71.4 \%$ of IBD patients compared to $85.2 \%$ of 27 control patients [21]. One further study with 6 patients on immunosuppressive therapy showed a lower antibody response to COVID-19 vaccination [22].

Sixty-five (90.3\%) patients in the IBD group reported taking immunomodulatory therapy $(27 / 37.5 \%)$ were treated with TNF-alpha blockers, vedolizumab was used in $19(26.4 \%)$ patients, $14(19.4 \%)$ had ustekinumab, 1 patient $(1.4 \%)$ was treated with methotrexate, and 1 patient (1.4\%) with azathioprine, respectively. Only 2 (2.8\%) patients were on steroid medication. 24 (33.3\%) had a therapy that included mesalazine. Disease duration (13.01 years, $\mathrm{SD}=10.6$ ) indicates that patients with long lasting more severe courses with the need for more intensive therapy were included in our study. However, disease duration had no impact on antibody formation in our study.

Overall, the antibody response was highly effective and prevalent in all patients with IBD after 2 vaccinations compared to the matched control group after full vaccination. The mean antibody levels for all IBD patients were $1,257.1 \mathrm{U} / \mathrm{mL}$ in males and $1,500.15 \mathrm{U} / \mathrm{mL}$ in female patients. In general, slightly decreased antibody response compared to the healthy control group could be detected. There was no vaccination failure in the IBD group. Stable antibody titers were reached in patients treated with either mesalazine, TNF-alpha blocker, integrin inhibitors, or interleukin inhibitors. As in published data described above, antibody levels did differ from controls while receiving immunomodulatory therapies.

After the first vaccination, stable antibody responses were measured for all vaccinations used in IBD patients: with BioNTech/Pfizer, the antibody titer was $1,375.76 \mathrm{U} /$ $\mathrm{mL}$ (SD 1,109.626); with Moderna, the titer was 1,349.5 U/ 
mL (SD 1,627.053) and with AstraZeneca the titer was $1,432.16 \mathrm{U} / \mathrm{mL}$ (SD 1,236.874). Overall, there was no significant difference in antibody levels between the vaccines used in our study ( $p$ value 0.904 ). The antibody levels after second vaccination were $1,434.62 \mathrm{U} / \mathrm{mL}$ (SD 1,108.2), 1,924.75 U/mL (SD 1,150.5), and 48.2 U/mL for BioNTech/ Pfizer, Moderna, and AstraZeneca, respectively. Again, no significant differences between the individual vaccines could be detected ( $p$ value 0.332 ). Since all employees in the control group received BioNTech/Pfizer exclusively for both vaccinations, data comparison of patients with Moderna or AstraZeneca vaccines was not possible.

Whether patients had UC or CD did not affect the level of antibody titers ( $p$ value 0.417 ). Sex did not influence antibody titer either, although female patients had higher antibody titers than men. Currently, no official cutoff value has been established, which guarantees sufficient protection against COVID-19 [23]. Our in-house recommendations regard a value above 10 $15 \mathrm{U} / \mathrm{mL}$ as a sufficient response to the SARS-CoV-2 vaccination. All patients in the IBD group therefore reached sufficient antibody response according to our guidelines.

There was a trend toward lower antibody titer in elderly patients. However, the differences were not statistically significant. This is in accordance with published data where lower rates of antibody responses in immunocompetent elderly patients are described after 2 doses of vaccination [24, 25]. This patient population might be of particular interest in tight monitoring of antibody levels over time to early detect patients with a need of re-vaccination. In general, all patients in the IBD (48.4 years, SD 15.2) and control group (46.3 years, SD 12.4) were younger than 60 years. Further studies are necessary to address the course of antibody titer over time in elderly patients with IBD.

There was a significant correlation between antibody response and time or days since the first vaccination with stronger correlations within the IBD group $(p<0.05)$ and lower within the control group $(p<0.01)$. However, the days between vaccination 1 and 2 had no impact on antibody response in both groups. We found a trend to higher antibody responses in patients with IBD who had a longer time to the last medication applied.

In the IBD patients, side effects after the first vaccination were higher compared to the control group, including muscle pain, pain at injection site, and fatigue. The opposite occurred after the second vaccination, when side effects were described as higher in the control group with similar leading symptoms after the first vaccination. In our study cohort, no further adverse events occurred, es- pecially in patients with IBD compared to data described in current literature $[4,26]$. But only a small part of patients in both groups responded to this issue in the questionnaire provided.

A limitation of our study is that not all patients could have blood drawn at a predefined given time. Blood samples were taken at different times after first or second vaccination. Furthermore, compared to the healthy control group, some patients in the IBD group received a different vaccine for first, second, or both vaccinations. The aim of the evaluation was to demonstrate an immune response in immunosuppressed patients with IBD independent of the vaccine applied.

In addition, it must be mentioned that the completed questionnaires were filled out retroactively by the patients themselves. A subjective assessment and reevaluation by medical staff did not take place again. So, side effects after first and second vaccination were not recorded in all patients. Due to laboratory capacities, the determination of antibody values above 2,500 U/ $\mathrm{mL}$ with further titrations was not possible and levels are limited to this value.

In conclusion, our study could show a $100 \%$ antibody response to vaccination against COVID-19 in patients with IBD and immunomodulatory therapies after 2 vaccinations. Antibody response was high in IBD patients even after the first vaccination - however, antibody levels were lower in IBD patients compared to controls. Overall, vaccination was well tolerated and no further or new adverse events were detected in IBD patients compared to healthy controls. There was a trend to reduced immune response in elderly patients; however, this was not significant. Disease duration and concomitant immunomodulatory medical therapy (TNF-alpha blockers, interleukin inhibitors, integrin inhibitors, methotrexate, or azathioprine) had no impact on the immune response. However, the time between the last medication given and the vaccination seems to have a positive effect on antibody response in IBD patients. Further evaluation of antibody titers over time is mandatory to detect early the need for re-vaccination in patients with IBD.

\section{Statement of Ethics}

The study was performed at the University Hospital Augsburg, Germany, in accordance with the Declaration of Helsinki and Good Clinical Practice. In addition, the study was approved by the Ethics Committee of the Ludwig-Maximilians-University in Munich, Germany (21-0693). Patients were included after given written informed consent. 


\section{Conflict of Interest Statement}

On behalf of all authors, the corresponding authors state that there is no conflict of interest.

\section{Funding Sources}

No funding was received for this study.

\section{Author Contributions}

J.M.C. and E.S. both equally wrote the main manuscript text and were responsible for acquisition, data and patient recruitment, data analysis and manuscript preparation. S.N., A.E., and C.F. were responsible for patient recruitment and manuscript reviewing. A.M. was responsible for statistical analysis and manuscript reviewing. C.R. and H.M. were responsible for manuscript reviewing.

\section{Data Availability Statement}

All data generated and analyzed during this study are included in this article and its online supplementary material. Further inquiries can be directed to the corresponding authors.

\section{References}

1 World Health Organization. Novel coronavirus (2019-nCoV) - situation report; 2020.

2 https://covid19.who.int/region/euro/country/de.

3 Papa A, Gasbarrini A, Lopetuso LR. Winter is coming and COVID-19 vaccine is available! The role of gastroenterologist in increasing COVID-19 vaccine acceptability among IBD patients. Gastroenterology. 2021;161(1):368-9.

4 Voysey M, Clemens SAC, Madhi SA, Weckx LY, Folegatti PM, Aley PK, et al. Safety and efficacy of the ChAdOx1 nCoV-19 vaccine (AZD1222) against SARS-CoV-2: an interim analysis of four randomised controlled trials in Brazil, South Africa, and the UK. Lancet. 2021;397(10269):99-111.

5 Sadoff J, Gray G, Vandebosch A, Cárdenas V, Shukarev G, Grinsztejn B, et al. Safety and efficacy of single-dose Ad26.COV2.S vaccine against Covid-19. N Engl J Med. 2021;384(23): 2187-201.

6 Magro F, Rahier JF, Abreu C, MacMahon E, Hart A, van der Woude CJ, et al. Inflammatory bowel disease management during the COVID-19 outbreak: the ten do's and don'ts from the ECCO-COVID taskforce. J Crohns Colitis. 2020;14:S798-806.

7 Allocca M, Fiorino G, Zallot C, Furfaro F, Gilardi $\mathrm{D}$, Radice $\mathrm{S}$, et al. Incidence and patterns of COVID-19 among inflammatory bowel disease patients from the nancy and milan cohorts. Clin Gastroenterol Hepatol. 2020;18:2134-5.

8 Stallmach A, Sturm A, Blumenstein I, Helwig U, Koletzko S, Lynen P, et al. (Addendum to S3-guidelines Crohn's disease and ulcerative colitis: management of patients with inflammatory bowel disease in the COVID-19 pandemic open questions and answers). Z Gastroenterol. 2020;58:e367.

9 Allocca M, Chaparro M, Gonzalez HA, Bosca-Watts MM, Palmela C, D'Amico F, et al. Patients with inflammatory bowel disease are not at increased risk of COVID-19: a large multinational cohort study. J Clin Med. 2020; 9(11):3533.

10 Siegel CA, Melmed GY, McGovern DP, Rai V, Krammer F, Rubin DT, et al. SARS-CoV-2 vaccination for patients with inflammatory bowel diseases: recommendations from an international consensus meeting. Gut. 2021; 70(4):635-40.

11 Alexander JL, Moran GW, Gaya DR, Raine T, Hart A, Kennedy NA, et al. SARS-CoV-2 vaccination for patients with inflammatory bowel disease: a British society of gastroenterology inflammatory bowel disease section and IBD clinical research group position statement. Lancet Gastroenterol Hepatol. 2021; 6(3):218-24.

12 Wellens J, Colombel JF, Satsangi JJ, Wong SY. SARS-CoV-2 vaccination in IBD: past lessons, current evidence, and future challenges. Crohns Colitis. 2021;15(8):1376-86.

13 Malipiero G, Moratto A, Infantino M, D’Agaro P, Piscianz E, Manfredi M, et al. Assessment of humoral and cellular immunity induced by the BNT162b2 SARS-CoV-2 vaccine in healthcare workers, elderly people, and immunosuppressed patients with autoimmune disease. Immunol Res. 2021;69(6):576.

14 Kennedy NA, Goodhand JR, Bewshea C, Nice $\mathrm{R}$, Chee D, Lin S, et al. Anti-SARS-CoV-2 antibody responses are attenuated in patients with IBD treated with infliximab. Gut. 2021; 70(5):865-75.

15 Kennedy NA, Lin S, Goodhand JR, Chanchlani N, Hamilton B, Bewshea C, et al. Infliximab is associated with attenuated immunogenicity to BNT162b2 and ChAdOx1 nCoV19 SARS-CoV-2 vaccines in patients with IBD. Gut. 2021;70(10):1884.

16 Fiorino G, Peyrin-Biroulet L, Naccarato P, Szabò $\mathrm{H}$, Sociale OR, Vetrano $\mathrm{S}$, et al. Effects of immunosuppression on immune response to pneumococcal vaccine in inflammatory bowel disease: a prospective study. Inflamm Bowel Dis. 2012;18:1042-7.

17 Hagihara Y, Ohfuji S, Watanabe K, Yamagami $\mathrm{H}$, Fukushima W, Maeda K, et al. Infliximab and/or immunomodulators inhibit immune responses to trivalent influenza vaccination in adults with inflammatory bowel disease. J Crohns Colitis. 2014;8:223-33.
18 Cullen G, Bader C, Korzenik JR, Sands BE. Serological response to the $2009 \mathrm{H} 1 \mathrm{~N} 1 \mathrm{influ}-$ enza vaccination in patients with inflammatory bowel disease. Gut. 2012;61:385-91.

19 Ben-Tov A, Banon T, Chodick G, Kariv R, Assa A, Gazit S. BNT162b2 messenger RNA COVID-19 vaccine effectiveness in patients with inflammatory bowel disease: preliminary real-world data during mass vaccination campaign. Gastroenterology. 2021;161(5):1715.

20 Wong SY, Dixon R, Martinez P, VGnjatic S, Colombel JF, Cadwell K. Serologic response to messenger RNA coronavirus disease 2019 vaccines in inflammatory bowel disease patients receiving biologic therapies. Gastroenterology. 2021;161:715-8.

21 Reuken PA, Andreas N, Grunert PC, Glöckner S, Kamradt T, Stallmach A. T cell response after SARS-CoV-2 vaccination in immunocompromised patients with inflammatory bowel disease. J Crohns Colitis. 2021.

22 Michos A, Tatsi EB, Filippatos F, Dellis C, Koukou D, Efthymiou V, et al. Association of total and neutralizing SARS-CoV-2 spike -receptor binding domain antibodies with epidemiological and clinical characteristics after immunization with the 1 (st) and 2(nd) doses of the BNT162b2 vaccine. Vaccine. 2021;39:5963-7.

23 Melgaco JG, Azamor T, Ano Bom APD. Protective immunity after COVID-19 has been questioned: what can we do without SARS-CoV-2IgG detection? Cell Immunol. 2020;353:104114.

24 Karamese M, Tutuncu EE. The effectiveness of inactivated SARS-CoV-2 vaccine (CoronaVac) on antibody response in participants aged 65 years and older. J Med Virol. 2021;94(1):173.

25 Collier DA, Ferreira IATM, Kotagiri P, Datir RP, Lim EY, Touizer E, et al. Age-related immune response heterogeneity to SARS-CoV-2 vaccine BNT162b2. Nature. 2021;596(7872):417-22.

26 Botwin GJ, Li D, Figueiredo J, Cheng S, Braun J, McGovern DPB, et al. Adverse events following SARS-CoV-2 mRNA vaccination among patients with inflammatory bowel disease. Am J Gastroenterol. 2021; 116(8):1746-51. 